\title{
SMOOTH PARTICLE APPLIED MECHANICS
}

\author{
Wm. G. Hoover, C. G. Hoover, O. Kum, and V. M. Castillo \\ Department of Applied Science \\ University of California at Davis-Livermore \\ and \\ Lawrence Livermore National Laboratory \\ Livermore, California 94551-7808
}

\author{
H. A. Posch \\ Institute for Experimental Physics \\ University of Vienna \\ A-1090 Vienna, Austria \\ S. Hess \\ Institute for Theoretical Physics \\ Technical University of Berlin \\ D-10623 Berlin, Germany
}

\begin{abstract}
Smooth Particle Applied Mechanics provides a novel method for solving the basic equations of continuum mechanics. The method is simple to implement, very stable, and applicable to a variety of far-from-equilibrium situations. It provides an interesting bridge between atomistic and continuum simulations. Here we describe our general explorations of the method, emphasizing recent results for the Rayleigh-Benard problem, a heat conducting flow driven by a convective instability.
\end{abstract}

\section{Introduction}

Computer experiments, both microscopic and macroscopic, are now sufficiently detailed to compete with real laboratory experiments as sources of reliable data. But microscopic molecular dynamics is limited to relatively short lengths and times. And macroscopic finite-difference or finite-element simulations are not only relatively complex, they are also plagued with numerical instabilities. A simple intermediate technique, Smooth Particle Applied Mechanics, "SPAM", has the simplicity and stability of molecular dynamics, but can be used to solve the continuum equations for relatively large space and time scales. The method is quite promising and deserves intensive investigation. Here we give a brief description of the method and then report on recent work exploring its applicability to relatively simple nonequilibrium fluid problems, in both two and three space dimensions. For literature references to the Smooth Particle approach the interested reader should consult our recent articles in the April and November 1995 issues of Physical Review E as well as forthcoming work in Physica A. 


\section{Smooth Particle Applied Mechanics}

It is an appealing idea to solve continuum problems in a way that parallels atomistic solutions, by using representative particles. That is what the smoothparticle approach does. There are two ideas underlying this approach to continuum problems. First, all the continuum properties-density, velocity, energy, stress, and heat flux, for instance-are to be repesented in space as very smooth interpolations of the same quantities defined on a discrete irregular grid of particles. Second, the grid particles themselves are to move, exchanging momentum and energy with immediately surrounding material according to local divergences of a stress tensor and heat flux vector based on continuum constitutive relations.

In 1977 Lucy and Monaghan independently discovered a practical scheme for interpolating on a moving grid, and for exchanging momentum and energy. They represented the spatial influence of each moving particle according to a normalized short-ranged weight function $w(r)$, with at least two continuous derivatives. The simplest example of such a weight function is Lucy's. Normalized in three dimensions, and with a range $h$, this function is:

$$
w_{\text {Lucy }}(r)=\left(105 / 16 \pi h^{3}\right)[1+3(r / h)][1-(r / h)]^{3} ; r<h .
$$

At any point in space, $r$, local values of the mass, momentum, and energy densities are to be calculated by summing the contributions of nearby particles. The local mass density $\rho_{r}$ for instance, is a superposition of contributions from every particle lying within the range $h$ of the point in question. The mass density at a point $i$ is similarly given by a special case of this same definition:

$$
\rho_{r}=m \sum_{j} w_{r j} ; \rho_{i}=m \sum_{j} w\left(r_{i j}\right)=m \sum_{j} w_{i j} .
$$

In such smooth-particle pair sums a typical smooth particle interacts with perhaps 40 of its neighbors. The very smooth character of $w$, with $\nabla w$ and $\nabla \nabla w$ continuous, then guarantees the resulting continuity of first and second spatial derivatives, such as $\nabla \rho, \nabla^{2} T$, and $\nabla \cdot \sigma$.

The smooth-particle form of the continuum equation of motion, $\rho \ddot{r}=\nabla \cdot \sigma$, likewise illustrates the simplicity of the gradient operation using smooth particles. The divergence of the stress tensor, evaluated at the location of particle $i$, for instance, becomes a sum of individual particle stress tensors multiplied by weight gradients for all particles within range of particle $i$ :

$$
\ddot{r}_{i}=m \sum\left[\left(\sigma / \rho^{2}\right)_{i}+\left(\sigma / \rho^{2}\right)_{j}\right] \cdot \nabla w_{i j} .
$$

Once initial conditions have been specified and boundary conditions implemented, usually by defining special boundary or image particles, relatively general nonequilibrium flows can be simulated and analyzed. We discuss some aspects of these flows in the following section. 


\section{General Aspects of SPAM}

Despite a close resemblance, SPAM is considerably more complex than is molecular dynamics. Computed continuum flows depend not only on the number of smooth particles used to represent the flow, but also on the form and range of the weight function. Our flows using Monaghan's weight function, for instance, exhibited a strong tendency for particles to move in pairs. Flows with Lucy's weight function did not show this behavior. Flows represented with a relatively short-ranged high-amplitude weighting function had a tendency toward artificial freezing, in a way fully consistent with Lindemann's law, which describes the freezing of atomistic fluids.

It is relatively straightforward to formulate smooth-particle equations so as to conserve mass, momentum, and energy exactly. Similarly, velocity and temperature gradients can be represented in such a way that particles with the same velocity or the same temperature make no contribution to the corresponding gradients. It is not so straightforward to conserve angular momentum, though the lack of conservation becomes rapidly less serious as the number of particles is increased. It is likewise not straightforward to treat boundaries, especially material interfaces. Mirror boundaries, in which external reflected-image particles are given specified velocity and temperature values, work well so long as the boundaries being represented are fixed in space. The smooth-particle form of the continuum variables also makes it particularly easy to change the number or shape of the particles during a simulation, and to evaluate the values of the flow variables on a regular grid, as is required for a fast fourier transform.

Smooth particles exhibit a curious form of Boltzmann's reversibility paradox. For the special case of an ideal gas with $P \propto \rho^{2}$ the smooth particle trajectories are isomorphic to molecular dynamics trajectories, with the smooth-particle weight function playing the role of a molecular-dynamics pair potential. The viscosities and thermal conductivity associated with the molecular dynamics must then necessarily correspond to artificial viscosities and artificial conductivity in the continuum simulations. The artificial numerical shear viscosity, and probably also the bulk viscosity and heat conductivity, has been found to vanish very rapidly with increasing numbers of particles, as $N^{-2}$ in two dimensions. This suggests that the smooth-particle method may be useful in simulating flows at high Reynolds number.

The isomorphism between smooth particle trajectories and atomistic ones has been borne out by the resemblance of smooth-particle Lyapunov spectra to those found in molecular dynamics simulations. No doubt the multifractal phase-space attractors which characterize nonequilibrium atomistic flows, can develop into disjoint attractors, each corresponding to a particular stationary flow, in the continuum limit. Figure 1 illustrates the Rayleigh-Bénard instability of a fluid heated from below in a gravitational field. The Figure shows three 
different flows, all for exactly the same external boundary conditions, from regular grid-based solutions of the Navier-Stokes equations. It appears that at least the two-roll and four-roll flows are stable stationary solutions of the continuum equations. The relative stabilities of coexisting stationary flows can only be evaluated empirically. The energies, entropy productions, and infinitesimal growth rates for such flows have all been studied as a part of Vic Castillo's dissertation work at the Department of Applied Science. In the following section we describe our investigation of such flows using SPAM.

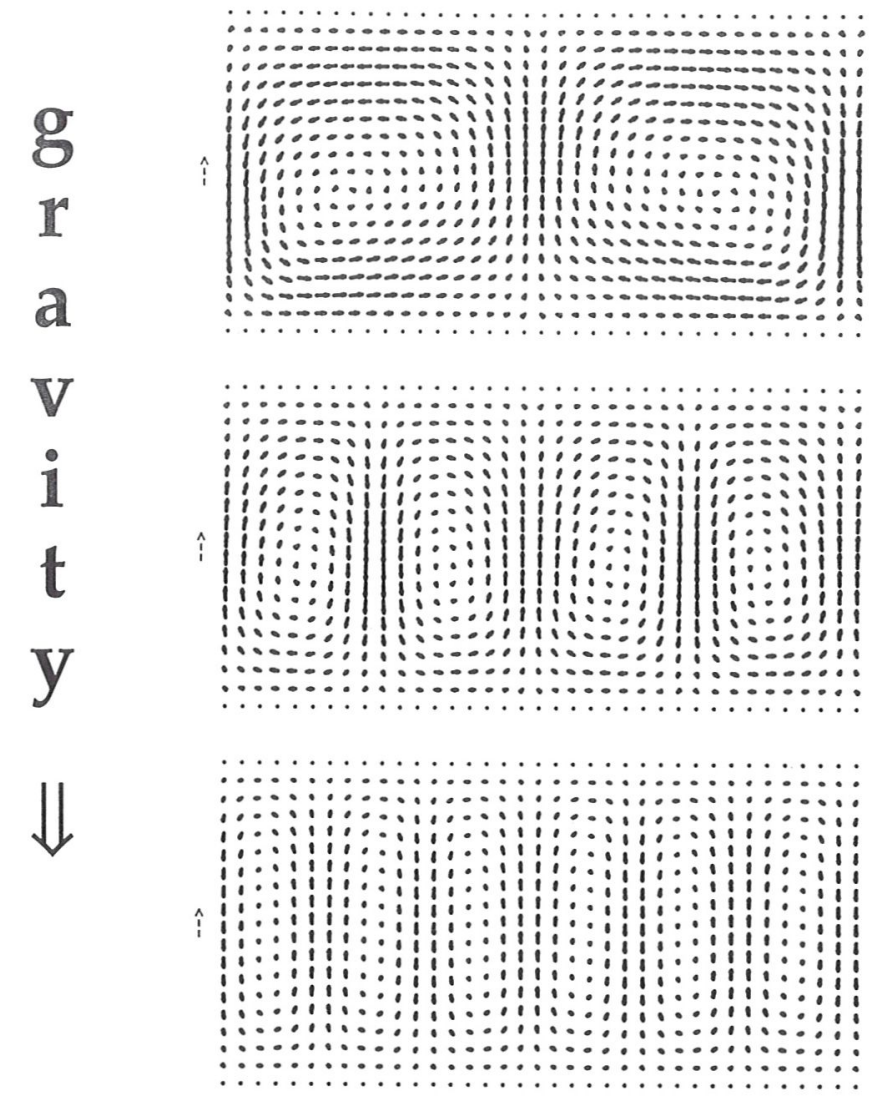

\section{Rayleigh-Bénard Roll Patterns $P=\rho k T=\rho e ; R a=40000 ; \operatorname{Pr}=1$}

Figure 1. Two-roll, four-roll, and six-roll solutions of the full continuum equations for a two-dimensional ideal gas with $\mathrm{Ra}=40000$ and $\mathrm{Pr}=1$. The two- and four-roll motions are stationary within computational double-precision accuracy. 


\section{Rayleigh - Benard Instability}

Oyeon Kum's 1995 doctoral research in the Department of Applied Science included a thorough assessment of SPAM's usefulness in solving the full RayleighBenard problem in two space dimensions. He emphasized flows in a convection cell, periodic in the horizontal direction and with a horizontal-to-vertical aspect ratio of two. Chandrasekhar's analysis of a Boussinesq fluid in this same geometry predicts convection at Rayleigh numbers exceeding 1708. We compared fully-converged conventional solutions of the complete nonlinear fluid equations with Kum and Hoover's independent SPAM simulations, as well as parallel solutions generated by Harald Posch. We found that SPAM reproduced both transient and stationary flows. Velocity errors of a few percent were typical of the smooth-particle simulations, which used only a few thousand particles.

Steady two-dimensional simulations can save computer time by providing initial conditions for corresponding three-dimensional flows. For the two- and three-dimensional simulations to correspond, it is necessary to use the same equilibrium equation of state in both cases. The shear and bulk viscosities have to be treated differently in the two cases. A three-dimensional fluid with no bulk viscosity has the following constitutive relation:

$$
\sigma_{x x}=\sigma_{e q}+(\eta / 3)\left(4 \dot{\epsilon}_{x x}-2 \dot{\epsilon}_{y y}-2 \dot{\epsilon}_{z z}\right)+0\left(\dot{\epsilon}_{x x}+\dot{\epsilon}_{y y}+\dot{\epsilon}_{z z}\right) ; \sigma_{x y}=\eta \dot{\epsilon}_{x y} .
$$

In plane strain, $\sigma_{x x}$ does not correspond exactly to the same stress component for a two-dimensional fluid with shear viscosity alone. In order for the two- and three-dimensional stresses to correspond, the two-dimensional fluid requires an additional bulk viscosity equal to one-third the shear, $\eta_{v}=\eta / 3$ :

$$
\sigma_{x x}=\sigma_{e q}+\eta\left(\dot{\epsilon}_{x x}-\dot{\epsilon}_{y y}\right)+\eta_{v}\left(\dot{\epsilon}_{x x}+\dot{\epsilon}_{y y}\right) ; \sigma_{x y}=\eta \dot{\epsilon}_{x y} .
$$

The numerical effect of this additional bulk viscosity turns out to be rather small. We checked it with accurate simulations of ideal-gas Rayleigh-Bénard flow using a regular finite-difference grid. At a Rayleigh number of 3600, the flow velocity increased by about one part in 1000 when the two-dimensional bulk viscosity was set equal to zero rather than to $\eta / 3$. It is interesting, and possibly significant, that taking a two-dimensional reference system with vanishing bulk viscosity would require a negative bulk viscosity in three dimensions, leading to catastrophic instabilities.

Three-dimensional flow simulations still strain the capabilities of serial machines. And tens of thousands of particles are required in order to avoid the serious errors associated with artificial freezing. Simulations with 36 X 18 X $36=$ 23328 smooth particles are a convenient size for presentday work stations. These three-dimensional simulations reveal considerable topological complexity. The simple two-roll structure, discussed by Chandrasekhar and shown in both Figures, was observed by us, in the three-dimensional SPAM simulations of Figure 2, 

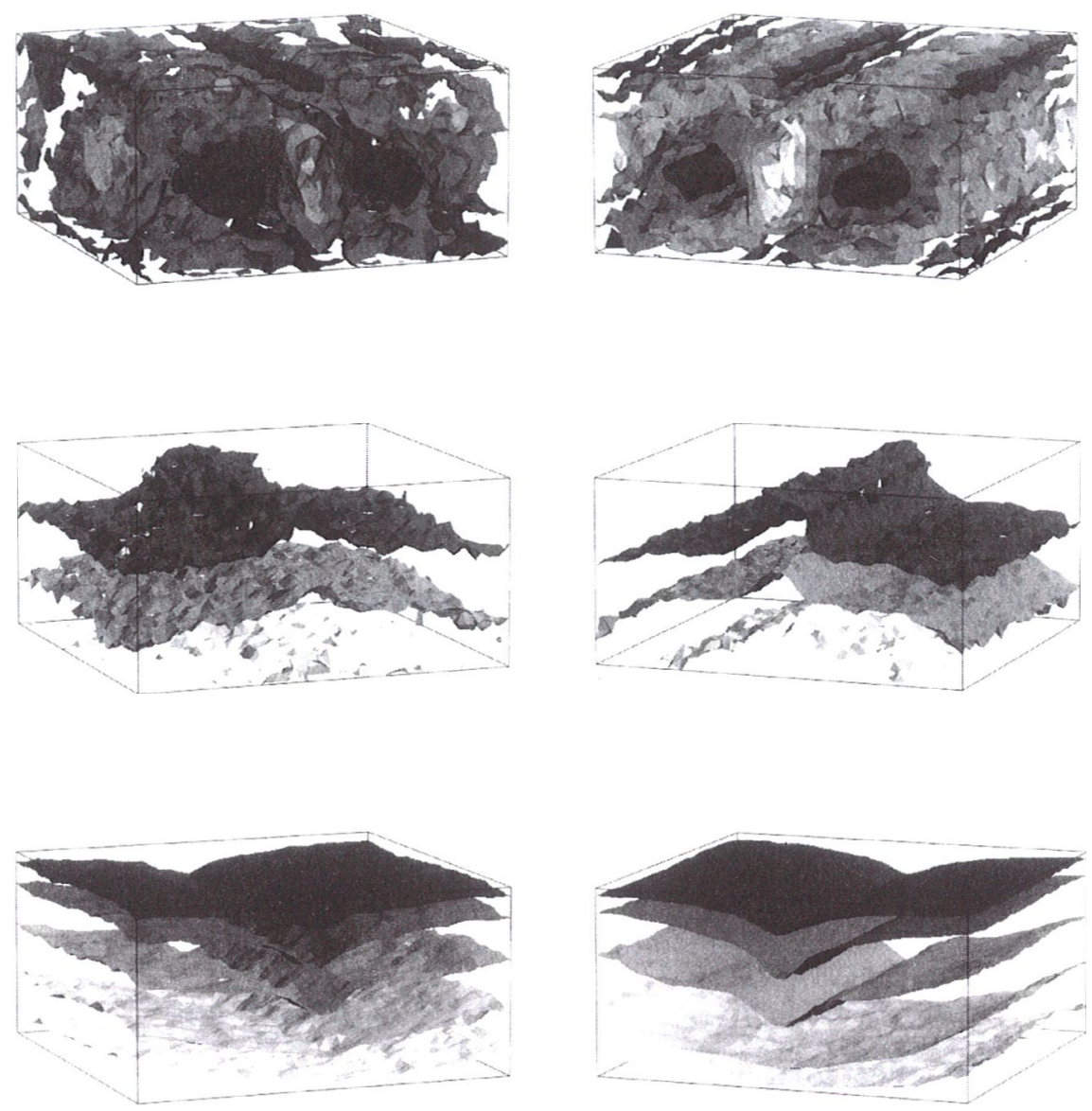

Figure 2. Smooth particle flows for a three-dimensional ideal gas, with $P=\rho k T=\rho e$, $\mathrm{Ra}=40000$, and $\operatorname{Pr}=1$. These solutions all used $36 \times 18 \times 36=23328$ smooth particles. The weighting functions are Monaghan's in both cases, with ranges $h$ equal to $2.50(\mathrm{~V} / \mathrm{N})^{1 / 3} \quad$ (right) and $2.75(\mathrm{~V} / \mathrm{N})^{1 / 3}$ (left). From top to bottom the surfaces represent particle speed, density, and temperature. The bottom-to-top temperature ratio is three. The flow at the center is downward. 
to transform to a rotated configuration, rotated ninety degrees about the vertical axis, suggesting also the stability of narrower intermediate rolls. Likewise, continuum simulations, in two dimensions and in three, starting with random velocities, were observed to form both two-roll solutions and four-roll solutions, as shown in Figure 1. with also an intermediate stationary two-roll state, in three dimensions, with the rolls rotated forty five degrees. Just as seems to be the case experimentally, a variety of continuum solutions can coexist. All these solutions have exactly the same boundary conditions. We emphasize that this degeneracy applies to solutions of the full continuum equations, without any simplifying assumptions or approximations.

Solutions of the Rayleigh-Bénard problem can be characterized by the dimensionless Rayleigh and Prandtl Numbers,

$$
R a \equiv g H^{3} /\left(\nu D_{T}\right) \equiv k \Delta T(H / \nu)\left(H / D_{T}\right) ; \quad \operatorname{Pr} \equiv \nu / D_{T}
$$

where $g$ is the gravitational acceleration; the system height is $H$; $\nu$ and $D_{T}$ are the kinematic viscosity and thermal diffusivity, $\nu=\eta / \rho$ and $\mathrm{D} \mathrm{j}=\kappa /\left(\rho c_{V}\right)$. We have used a variety of initial conditions. If the nodal velocities are chosen randomly, the corresponding kinetic energy dies rapidly except for the contributions of unstable modes. These modes grow, exponentially fast when small, with the most successful mode winning out, and finally approaching a finite-amplitude stationary limit cycle, of the type shown in Figure 1.

Particle speeds, densities, and temperatures are shown in Figure 2 for two different ranges of Monaghan's weighting function. Both simulations were carried out at an intermediate Rayleigh number of 40000 , about 23 times the critical value for two-roll convection, and perhaps half the value required for turbulent flow. The computed smooth-particle solutions of Figure 2 are much less regular than the stationary continuum simulations, due to the presence of fluctuations. The two-dimensional continuum simulations of Figure 1, show that even regular grids can provide a multiplicity of solutions. In the smooth particle case the fluctuations never cease, so that, at least in principle, the possibility of macroscopic flow-pattern changes remains, even at long times. In many situations the convective rolls and cells can come and go, and change orientation, in times which are quite long relative to a typical roll rotation time. Though the stability of such two-roll patterns is well known in simulations, with both molecular dynamics and smooth particle applied mechanics, the four-roll and six-roll solutions reported in the present work were a surprise to us, and are undergoing continuing investigation.

Our two-dimensional results at a Rayleigh number of 40000 are shown in the table. The six-roll solution has the lowest energy, the four-roll solution has the highest growth rate, and the two-roll solution has the largest entropy production and kinetic energy. Evidently there is no way to characterize the relative stability of the various solutions of the continuum equations. 


\begin{tabular}{|c|c|c|c|c|c|}
\hline Rolls & $K_{x} / N m$ & $K_{y} / N m$ & $E / N m$ & $Q_{\text {boundary }}$ & $W / \tau$ \\
\hline 2 & 0.00373 & 0.00357 & 1.014 & 0.0120 & 1.42 \\
4 & 0.001139 & 0.00410 & 1.018 & 0.0118 & 1.70 \\
6 & 0.000274 & 0.00226 & 1.012 & 0.0106 & 1.25 \\
\hline
\end{tabular}

Table 1. The horizontal and vertical contributions to the kinetic energy per unit mass, the internal energy per unit mass, the boundary heat flux, and the small-amplitude growth rate of the kinetic energy, multiplied by the system width $W$ are given for a series of fully-converged solutions at a Rayleigh umber of 40000. Two-roll, four-roll, and six-roll solutions are compared. $W=2 H . \quad N=2 H^{2}$. In the continuum limit energies vary as $N$, traversal times, diffusion times, and dissipation rates as $N^{1} l^{2} \propto H$.

\section{Summary}

SPAM provides a simple and robust approach to a variety of continuum flow problems. It rewards investigators with a host of surprises and paradoxes while at the same time enhancing our understanding of the link between the atomistic and continuum approaches to flow simulation. 